\title{
Oral pregnancy tumour and its surgical management: A case report
}

\author{
Parul Chhabra ${ }^{1, *}$, Pujit Chhabra $^{2}$, Rajnish Verma ${ }^{3}$, Vinod $^{4}$, Kashika Sharma $^{5}$ \\ ${ }^{1}$ MDS Periodontist, ${ }^{2,5}$ PG Student Orthodontist, ${ }^{3,4} \mathrm{PG}$ Student Periodontist, ${ }^{\mathbf{1 , 3}, \mathbf{4}}$ Dept. of Periodontics, ${ }^{\mathbf{2}, \mathbf{5}}$ Dept. of Orthodontics, Pandit \\ Bhagwat Dayal Sharma Post Graduate Institute of Medical Science, Rohtak, Haryana, India
}

*Corresponding Author:

Email: drparulc@gmail.com

\begin{abstract}
In oral cavity Pregnancy tumour is an exophytic tissue. It occurs after three months of pregnancy because of vascular effects of hormones during pregnancy. Clinically it appears as sessile or pedunculated exophytic mass, having bleeding tendency. It occurs in response to numerous factors like traumatic injury, low grade irritation, or hormonal changes. For its treatment various modalities are used like flash lamp pulsed dye laser, cryosurgery, Nd: YAG laser, intralesional injection of ethanol, corticosteroids, and sodium tetradecyl sulfate sclerotherapy, but still surgical excision is the treatment of preference.
\end{abstract}

Keywords: Pregnancy, Pyogenic granuloma, Tumour.

\section{Introduction}

During pregnancy changes in oral cavity are seen with hormonal changes (like pregnancy gingivitis and pregnancy Tumour) and certain systemic health issues may be seen (like Low birth weight baby, risk of preterm). Out of all Oral pregnancy Tumour (OPT) is a frequent pathology.

The OPT, also acknowledged as pyogenic granuloma (PG), granuloma pyogenicum or granuloma gravidarum, is a non-neoplastic, non-malignant lesion and one of the inflammatory hyperplasia (Gondivkar et al. 2010 Kamal et al. 2012). ${ }^{1}$

The first case of OPT was reported by Hullihen (1844) and the term pyogenic granuloma was given by Hartzell however the term is a misnomer because it is not a true granuloma and no pus is present. ${ }^{2}$ Blum in 1912 gave the term Pregnancy tumour. ${ }^{3}$ It shows an inflammatory response to plaque microorganisms. Occur in $2^{\text {nd }}$ and $3^{\text {rd }}$ trimester of pregnancy, but it may occur earlier. ${ }^{4}$

\section{Etiology}

It can occur in response to various stimuli, such as localized low-grade irritation, traumatic injury or hormonal changes - increased oestrogen and progesterone levels (hormonal changes induce an increased vascular permeability, which leads to gingival edema and an increased inflammatory response to dental plaque). ${ }^{4}$ The increased hormones increases the expression of angiogenic factors in inflamed tissue, and supresses apoptosis of granuloma cells to expand angiogenic effects (Yuan et al. 2002). ${ }^{1}$

\section{Clinical Features}

Overgrowth is usually generalized, but it can be more prominent interproximally. Overgrowth is bright red or magenta, soft, and friable that bleeds spontaneously or on slight provocation. The enlarged gingiva appears clinically as a distinct, mushroom-like, flattened spherical mass that extends from the free gingival margin or, interproximal gingiva with a sessile or pedunculated base. Surface appears smooth, glistening with numerous deep red, pinpoint markings. Radio graphically it does not invade the underlying bone. ${ }^{4}$

Ziskin and Ness (1946) presented a clinical classification of pregnancy gingivitis. ${ }^{5}$
1. Class I - bleeding gingiva with more or less no other changes

2. Class II - changes in interdental papilla-edema and swelling, a tendency to recur. Subsequent blunting of interdental papilla

3. Class III - involvement of the free gingival margin, which takes on the color and general appearance of a raspberry

4. Class IV - Generalized hypertrophic gingivitis

5. Class $\mathrm{V}$ - oral pregnancy Tumour.

\section{Case Report}

A 23-year-old woman in the 2nd trimester of pregnancy reported to the Department of Periodontology, JCD Dental College Sirsa with the chief complaint of gingival enlargement in mandibular right lingual region which bleed frequently and interfered with chewing food. On intraoral examination revealed a $1.5 \mathrm{~cm} \times 1 \mathrm{~cm}$ pedunculated exophytic mass covered by glistening red mucosa. The enlarged tissue was from first premolar region to the first molar region its consistency was soft on palpation, bleeding on palpation was present with no ulceration (Fig. 1). The patient was not capable to maintain oral hygiene in that area, because of gingival overgrowth, rest of oral cavity showed normal gingiva and satisfactory oral hygiene. A provisional diagnosis of pregnancy Tumour was made. Differential diagnosis of peripheral giant cell granuloma was made.

A. Radiographically no bone loss was observed.

Phase I incorporated Full mouth scaling after routine $\mathrm{CBC}$ and oral hygiene instructions were given. Oral prohylaxis resulted in the removal of plaque, calculus, and decrease in gingival inflammation. After decrease in inflammation, surgical excision was planned. 
Table 1: Clinical management of an oral pregnancy Tumour $(\mathrm{Opt}) \mathrm{.}^{1}$

\begin{tabular}{|l|l|}
\hline Clinical management strategies & $\begin{array}{l}\text { 1. Routine oral examination and treatment of existing } \\
\text { periodontal inflammation before conception } \\
\text { 2. Intergration of dental care into preconception counseling }\end{array}$ \\
\hline Prevention during pregnancy & $\begin{array}{l}\text { 1. Routine oral examinations and oral hygiene } \\
\text { 2. Removal of dental plaque } \\
\text { 3. Use of soft toothbrushes }\end{array}$ \\
\hline Diagnosis & If oral mass is present: suspect OPT, refer to dentist \\
\hline $\begin{array}{l}\text { Treatment } \\
\text { Small lesion, typical features and no } \\
\text { significant } \\
\text { functional or esthetic problems }\end{array}$ & $\begin{array}{l}\text { Excision after pregnancy, because they may recur during } \\
\text { pregnancy and can resolve spontaneously after delivery }\end{array}$ \\
\hline With pain and/or unsecure diagnosis & Surgical excision \\
\hline Prevention of recurrence & $\begin{array}{l}\text { 1. Excised down to the underlying periosteum } \\
\text { 2. Removal of predisposing irritant } \\
\text { 3. Careful oral prohylaxis and removal of dental plaque } \\
\text { 4. Use of soft toothbrushes }\end{array}$ \\
\hline
\end{tabular}

\section{Surgical Procedure}

Local anesthesia 1:80,0000 mandibular nerve block and lingual nerve block was administered. For excision of the enlargement scalpel was used. After excision, root planning and curettage of the site was done and normal saline was used for irrigation (Fig. 2) and postoperative instructions were given. The patient was recalled for examination after 10 days to see the healing and gingival tissue status. Early healing was uneventful. The patient was reinstructed for oral hygiene maintenance.

For histopathological examination the excised enlarged mass was delivered to laboratory (Fig. 3). $\mathrm{H}$ and E-stained section shows ulcerated stratified squamous epithelium with an underlying fibrovascular stroma with numerous capillaries, fibroblasts and areas of extravagated blood and infiltration of chronic inflammatory cell (Fig. 4). Based on a clinicopathological association, a final diagnosis of pregnancy Tumour was made. On 6 months regular follow up no recurrence has been observed.

\section{Discussion}

OPT develops in up to $5 \%$ of pregnancies in the $2^{\text {nd }} \& 3^{\text {rd }}$ trimester of pregnancy. ${ }^{2}$ Surgical excision is treatment of choice but it has a high recurrence rate of 3 $-23 \%$. To avoid recurrence, the lesion must be excised upto the underlying periosteum to remove the source of continuing irritation. ${ }^{7}$ Other treatment modalities include: flash lamp pumped-pulsed dye laser, cryosurgery and in cases of recurrent cases, intralesional injections of corticosteroids, ethanol, sodium tetradecyl sulphate. ${ }^{1}$ and to avoid occurrence and recurrence of a pregnancy Tumour regular oral prophylaxis dental plaque removal and use of soft toothbrushes plays a significant role. Now a days Nd:YAG laser is practiced because of the lesser bleeding as compared to other Surgical techniques. ${ }^{8}$
Increase in estrogen and progesterone levels in pregnancy changes normal oral flora to anaerobic flora with Prevotella intermedia predominance. Hormones act as a substitute for menadione (Vitamin $\mathrm{K}$ ) which is essential for the growth of $\mathrm{P}$. intermedia and coincide with gingival bleeding. ${ }^{9}$

In macrophage, estrogen enhance vascular endothelial growth factor (VEGF) production, which is associated to the progress of pregnancy Tumour. In pregnancy, Progesterone works as an immunosuppressant in the gingival tissues, inhibiting a rapid acute inflammatory reaction against plaque, but leading to amplified chronic tissue response, resulting an exaggerated appearance of inflammation clinically. ${ }^{2}$

O'Neil (1979) reported that in pregnancy there is suppression of immunity because of decrease in maternal T-lymphocytes. Which lead to exaggerated tissue response to altered flora. ${ }^{10}$

In the present case, gingival overgrowth was causing functional problem, patient CBC values were in normal range and with physician consent, and surgical management along with maintenance therapy was planned.

\section{Conclusion}

Oral hygiene instructions should be taught to every pregnant woman and encouraged that hygiene should not be neglected throughout pregnancy care. Patient should be informed it is a non-neoplastic enlargement.

Referral to a dentist by gynecologist is must to make the good diagnosis and initiate early treatment to avoid further complications such as severe bleeding. 


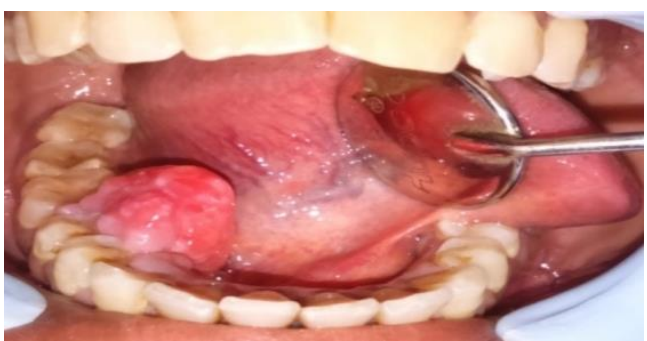

Fig. 1: Pre- operative photograph

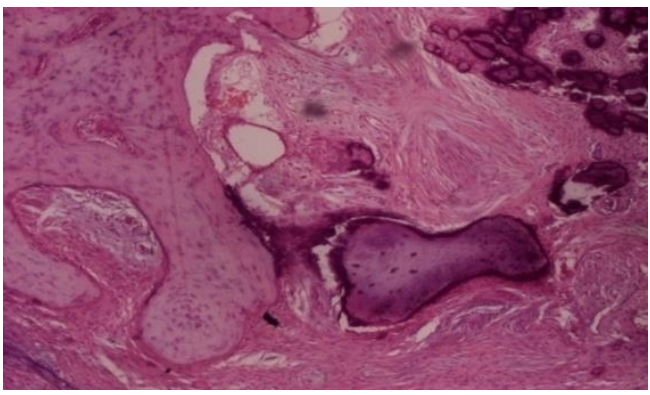

Fig. 2: Immediately after excision

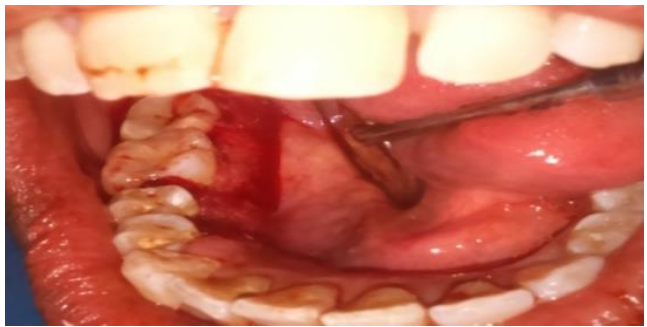

Fig. 3: Excised tissue

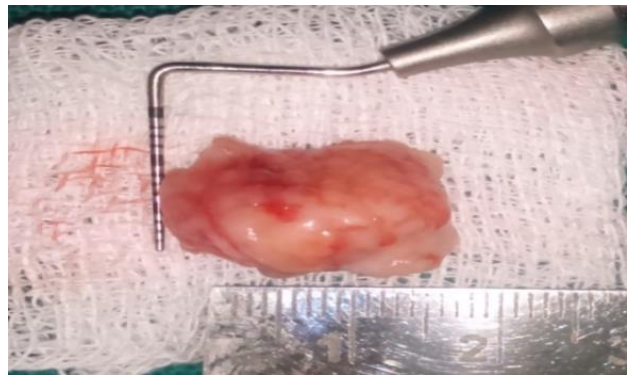

Fig. 4: Histopathological examination

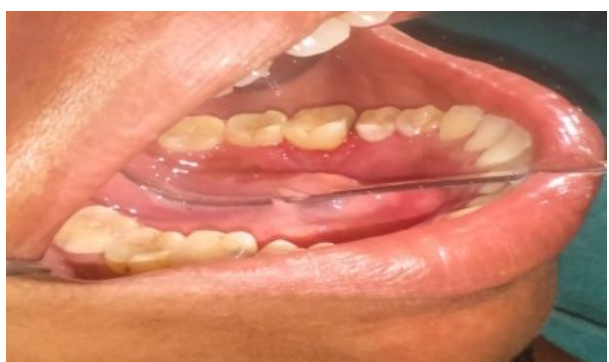

Fig. 5: Six months post-operative photograph (Indirect view in intraoral mirror)

\section{References}

1. Manegold-Brauer G, Brauer H.U. Oral pregnancy tumour: An update. $J$ of Obstet and Gynaecol. 2014;34:187-88.

2. Shailesh GM, Amol G, Revant C. Oral pregnancy Tumour. Contem Clin Dent. 2010;1(3).

3. Nisha S, Shivamallu AB, Hedge U, Oral Pregnancy Tumour. J of Dent and Allied Sciences. 2018;7(1).

4. Newmann MG, Takei HH, Klokkevold PR, Carranza FA. Carranza's Clinical Periodontology. $10^{\text {th }}$ Ed.

Philadelphia: W.B. Saunders Co. 2002;639-42.

5. Stark MM, Zarka FJ. Gingival Tumours of pregnancy; review of pregnancy Tumours and a report of two cases. Obstet Gynaecol. 1956;8:597-600.

6. Ojanotko-Harri AO, Harri MP, Hurttia HM, Sewon LA. Altered tissue metabolism of progesterone in pregnancy gingivitis and granuloma. J Clin Periodontol. 1991;18:262-66.

7. Neville BW, Damm DD, Allen CM, Bouquot JE. Oral and maxillofacial pathology. 2nd ed. Philadelphia: W.B Saunders.2002;437-95.

8. Powell JL, Bailey CL, Coopland AT, Otis CN, Frank JL, Meyer I. Nd: YAG laser excision of a giant gingival pyogenic granuloma of pregnancy. Lasers Surg Med. 1994;14:178-83.

9. Kornman KS, Loesche WJ. The subgingival microbial flora during pregnancy. J Periodontal Res. 1980;15:11122.

10. O’Neil TC. Maternal T-lymphocyte response and gingivitis in pregnancy. J Periodontol. 1979;50:178-78.

How to cite this article: Chhabra $\mathrm{P}$, Chhabra $\mathrm{P}$, Verma R, Vinod, Sharma K. Oral pregnancy tumour and its surgical management: A case report. Int $\mathbf{J}$ Periodontol Implantol. 2018;3(4):131-133. 